\title{
CT-based radiomics and machine learning for the prediction of myocardial ischemia: Toward increasing quantification
}

\author{
Andrew Lin, MBBS, BMedSci, ${ }^{a}$ and Damini Dey, $\mathrm{PhD}^{\mathrm{a}}$ \\ a Biomedical Imaging Research Institute, Cedars-Sinai Medical Center, Los Angeles, CA
}

Received Jun 18, 2020; accepted Jun 19, 2020

doi: 10.1007/s12350-020-02261-7

\section{See related article, pp. 262-274}

Current clinical interpretation of coronary computed tomography angiography (CTA) is limited to anatomical assessment of stenosis severity, ${ }^{1}$ which alone does not determine the hemodynamic significance of coronary lesions. ${ }^{2,3}$ The addition of static CT myocardial perfusion significantly increases the diagnostic performance of CTA for detecting flow-limiting coronary artery disease with stress single-photon emission computed tomography myocardial perfusion imaging (SPECT-MPI) as the reference standard. ${ }^{4}$ However, the clinical implementation of CT perfusion is limited by an increased radiation dose for patients and a lack of standardized scanning protocols. ${ }^{5}$ Visual assessment of perfusion deficits on standard coronary CTA images is also challenging due to the relatively poor contrast resolution. ${ }^{6}$ Radiomics is the process of extracting a large number of quantitative features from medical images to create big data in which each abnormality is characterized by hundreds of parameters indiscernible to the human eye. ${ }^{7}$ Computational techniques such as data mining and machine learning can then be used to identify new imaging patterns or biomarkers that associate with clinical features or outcomes. ${ }^{8}$ In cardiac magnetic

Funding Dr Andrew Lin and Dr Damini Dey are supported by a grant from the National Heart, Lung, and Blood Institute, USA [1R01HL133616].

Reprint requests: Damini Dey, PhD, Biomedical Imaging Research Institute, Cedars-Sinai Medical Center, 116 N Robertson Boulevard, Los Angeles, CA 90048; damini.dey@cshs.org

J Nucl Cardiol 2022;29:275-7.

$1071-3581 / \$ 34.00$

Copyright (c) 2020 American Society of Nuclear Cardiology. resonance imaging, radiomic texture analysis has been used for characterizing myocardial scar, ${ }^{9}$ distinguishing between acute and chronic infarction, ${ }^{10}$ and diagnosing myocarditis. ${ }^{11}$ The application of radiomics to coronary CTA has predominantly been in plaque analysis, where it has demonstrated superior accuracy to conventional qualitative and quantitative parameters for the identification of high-risk plaque. ${ }^{12,13}$ Few studies have performed CT-based radiomic analysis of cardiac structures such as the myocardium.

In this issue of Journal of Nuclear Cardiology, Shu et al. ${ }^{14}$ used machine learning to develop and validate a coronary CTA-derived radiomics nomogram for the prediction of chronic myocardial ischemia by SPECTMPI. They retrospectively studied patients who underwent both imaging modalities within a one-week interval, allocated into training $(\mathrm{n}=107)$ and testing $(n=47)$ cohorts. An independent cohort of 49 patients was used for external validation. Myocardial ischemia was defined as fixed or reversible perfusion defects on SPECT-MPI by visual assessment. Using CCTA images, a total of 378 textural radiomic parameters were calculated from three-dimensional myocardial segmentations, and feature dimensionality reduction was used to select 8 parameters which constituted a 'radiomics signature' for each patient. This was then input into a machine learning model along with clinical factors and stenosis severity grade to compute a 'radiomics nomogram'; essentially an individualized risk score for myocardial ischemia. The accuracy of the machine learned nomogram for predicting SPECT-MPI-determined ischemia was $0.839,0.832$, and 0.816 for the training, testing, and validation cohorts, respectively. Using decline curve 
analysis, the investigators showed good clinical net benefit in using the nomogram to predict ischemia in all three cohorts. In the entire study population, the radiomics nomogram (area under the receiver operator curve [AUC] 0.824) outperformed the radiomics signature (AUC 0.736, $P=.026$ ) and stenosis severity grade (AUC $0.708, P<.0001$ ) for the discrimination of myocardial ischemia.

While this represents a proof-of-concept study with small training and validation cohorts, Shu et al. ${ }^{14}$ are to be commended for their novel work in the field of cardiac CT radiomics. Given the challenges of CT perfusion and difficulties in evaluating myocardium on routine CTA, the investigators sought to provide a quantitative surrogate measure of the risk of myocardial ischemia. Radiomic analysis can be performed on standard coronary CTA images without the need for additional iodinated contrast or radiation exposure to the patient. The present study calculated only textural (gray level co-occurrence matrix and run-length matrix) radiomic parameters, with the aim of characterizing the heterogeneity of myocardial tissue via the spatial distribution of voxels. However, as conventional CT perfusion assessment relies on attenuation differences between normal and ischemic myocardium, ${ }^{5}$ it is likely that first-order (intensity-based) radiomic metrics would also be of value. Further, their CT-based radiomic nomogram was developed and validated using subjective visual, not quantitative, assessment of ischemia on SPECT-MPI as the reference standard. Importantly, a global 'radiomic signature', as a representative biomarker of ischemia anywhere in the myocardium, should be validated by quantitative measure of myocardial ischemia by SPECT or PET. ${ }^{15}$ Myocardial ischemia is also typically vessel-specific, with perfusion deficits localizing to the associated myocardial segments; this is true for both SPECT-MPI and CT perfusion. Future studies should also assess whether radiomic analysis according to myocardial vascular territories can predict vessel-specific quantitative measures of ischemia. Finally, the 'radiomic nomogram' in this study requires validation in larger, independent cohorts.

With the rapid generation of big data by cardiovascular imaging, there is a demand for increasingly sophisticated and efficient computational techniques to interpret these datasets. Advancements in radiomic feature extraction and machine learning methods will enable the identification of new quantitative imaging biomarkers that may enhance current coronary CTA assessment, by accurately predicting the risk myocardial ischemia or future adverse events.

\section{Disclosures}

The authors declare that they have no conflicts of interest.

\section{References}

1. Leipsic J, Abbara S, Achenbach S, Cury R, Earls JP, Mancini GJ, et al. SCCT guidelines for the interpretation and reporting of coronary CT angiography: A report of the Society of Cardiovascular Computed Tomography Guidelines Committee. J Cardiovasc Comput Tomogr 2014;8:342-58.

2. Gaemperli O, Schepis T, Valenta I, Koepfli P, Husmann L, Scheffel $\mathrm{H}$, et al. Functionally relevant coronary artery disease: Comparison of 64-section CT angiography with myocardial perfusion SPECT. Radiology 2008;248:414-23.

3. Meijboom WB, Van Mieghem CA, van Pelt N, Weustink A, Pugliese F, Mollet NR, et al. Comprehensive assessment of coronary artery stenoses: Computed tomography coronary angiography versus conventional coronary angiography and correlation with fractional flow reserve in patients with stable angina. J Am Coll Cardiol 2008;52:636-43.

4. Rochitte CE, George RT, Chen MY, Arbab-Zadeh A, Dewey M, Miller JM, et al. Computed tomography angiography and perfusion to assess coronary artery stenosis causing perfusion defects by single photon emission computed tomography: The CORE320 study. Eur Heart J 2014;35:1120-30.

5. Rossi A, Merkus D, Klotz E, Mollet N, de Feyter PJ, Krestin GP. Stress myocardial perfusion: Imaging with multidetector CT. Radiology 2014;270:25-46.

6. Danad I, Fayad ZA, Willemink MJ, Min JK. New applications of cardiac computed tomography: Dual-energy, spectral, and molecular CT imaging. JACC Cardiovasc Imaging 2015;8:710-23.

7. Kolossvary M, Kellermayer M, Merkely B, Maurovich-Horvat P. Cardiac computed tomography radiomics: A comprehensive review on radiomic techniques. J Thorac Imaging 2018;33:26-34.

8. Lin A, Kolossváry M, Išgum I, Maurovich-Horvat P, Slomka PJ, Dey D. Artificial intelligence: Improving the efficiency of cardiovascular imaging. Expert Rev Med Dev 2020. https://doi.org/ 10.1080/17434440.2020.1777855.

9. Kotu LP, Engan K, Skretting K, Måløy F, Ørn S, Woie L, Eftestøl T. Probability mapping of scarred myocardium using texture and intensity features in CMR images. BioMed Eng OnLine 2013;12:91.

10. Larroza A, Materka A, Lopez-Lereu MP, Monmeneu JV, Bodi V, Moratal D. Differentiation between acute and chronic myocardial infarction by means of texture analysis of late gadolinium enhancement and cine cardiac magnetic resonance imaging. Eur $\mathbf{J}$ Radiol 2017;92:78-83.

11. Baessler B, Luecke C, Lurz J, Klingel K, Roeder MV, Waha SD, et al. Cardiac MRI texture analysis of T1 and T2 maps in patients with infarctlike acute myocarditis. Radiology 2018;289:357-65.

12. Kolossváry M, Park J, Bang J-I, Zhang J, Lee JM, Paeng JC, et al. Identification of invasive and radionuclide imaging markers of 
coronary plaque vulnerability using radiomic analysis of coronary computed tomography angiography. Eur Heart $\mathrm{J}$ Cardiovasc Imaging 2019;20:1250-8.

13. Kolossvary M, Karady J, Szilveszter B, Kitslaar P, Hoffmann U, Merkely B, et al. Radiomic features are superior to conventional quantitative computed tomographic metrics to identify coronary plaques with napkin-ring sign. Circ Cardiovasc Imaging. 2017;10:e006843.

14. Shu Z, Cui S, Zhang Y, Xu Y, Huang S, Fu L, et al. Predicting chronic myocardial ischemia using CCTA-based radiomics machine learning nomogram. J Nucl Cardiol. 2020. https://doi.org/ 10.1007/s12350-020-02204-2.

15. Otaki Y, Betancur J, Sharir T, Hu L-H, Gransar H, Liang JX, et al. 5 -Year prognostic value of quantitative versus visual MPI in subtle perfusion defects. JACC Cardiovasc Imaging. 2020;13:774.

Publisher's Note Springer Nature remains neutral with regard to jurisdictional claims in published maps and institutional affiliations. 